\title{
Ultrasensitive Detection of Testosterone Using Microring Resonator with Molecularly Imprinted Polymers
}

\author{
Yangqing Chen ${ }^{1}$, Yong Liu ${ }^{1}$, Xiaodan Shen ${ }^{1}$, Zhimin Chang ${ }^{2}$, Longhua Tang ${ }^{1}$, Wen-Fei Dong ${ }^{2}$, \\ Mingyu $\mathrm{Li}^{1}{ }^{1, *}$ and Jian-Jun $\mathrm{He}^{1}$ \\ Received: 4 November 2015; Accepted: 11 December 2015; Published: 15 December 2015 \\ Academic Editor: Sandeep Kumar Vashist \\ 1 State Key Laboratory of Modern Optical Instrumentation, Zhejiang University, Hangzhou 310027, \\ Zhejiang, China; chenyangqing@ioe-zju.org (Y.C.); liuyong@ioe-zju.org (Y.L.); \\ 3110102785@zju.edu.cn (X.S.); lhtang@zju.edu.cn (L.T.); jjhe@zju.edu.cn (J.-J.H.) \\ 2 CAS Key Lab of Bio-Medical Diagnostics, Suzhou Institute of Biomedical Engineering and Technology, \\ Chinese Academy of Sciences, Suzhou 215163, Jiangsu, China; changzm@sibet.ac.cn (Z.C.); \\ wenfeidong@sibet.ac.cn (W.-F.D.) \\ * Correspondence: limy@zju.edu.cn; Tel.: +86-571-8795-3066
}

\begin{abstract}
We report ultrasensitive and highly selective detection of testosterone based on microring resonance sensor using molecularly imprinted polymers (MIP). A silicon-on-insulator (SOI) micoring resonator was modified by MIP films (MIPs) on a surface. The MIPs was synthesized by thermopolymerization using methacrylic acid as functional monomer and ethylene glycol dimethacrylate as crosslinking agent. The concentration of detected testosterone varies from $0.05 \mathrm{ng} / \mathrm{mL}$ to $10 \mathrm{ng} / \mathrm{mL}$. The detection limit reaches $48.7 \mathrm{pg} / \mathrm{mL}$. Ultrahigh sensitivity, good specificity and reproducibility have been demonstrated, indicating the great potential of making a cost effective and easy to operate lab-on-Chip and down scaling micro-fluidics devices in biosensing.
\end{abstract}

Keywords: Microring resonator; Molecularly imprinted polymers; Testosterone

\section{Introduction}

Testosterone is a special steroid hormone that acts as the primary androgenic hormone. It is mainly secreted through the reproductive organs, playing key roles in human health. The testosterone levels depend on age, the typical value for male adults is $2.01 \sim 7.50 \mathrm{ng} / \mathrm{mL}$ [1]. Low testosterone levels can lead to serious problems like underdeveloped genitalia, abnormalities in skeletal and muscle development, and diminished masculinity [2]. A precise detection of testosterone levels is thus important to the study of medical and sports endocrinology [3]. Traditional blood sampling is limited as the testosterone levels are closely related to serum and plasma free concentrations [4]. A non-invasive measurement in saliva is preferred, as the salivary testosterone mainly exists in the free form rather than bounding to sex hormone binding globulin. This method is further beneficial as it eliminates the need of specialist sampling equipment and allows for large quantities of acquisition. Currently, numerous efforts have been devoted to detect testosterone, including enzyme-linked immunosorbent assay (ELISA) techniques [5], radioimmunoassay (RIA) [6,7], high performance liquid chromatography (HPLC) [8], gas chromatography-mass spectrometer (GC-MS) [9] and liquid chromatography-mass spectrometry (LC-MS) [10]. These time-consuming procedures require specialized personnel and expensive instrumentation, limiting them from been more extensively used. A more easy-to-use, economical and rapid method is thus expected.

Microring resonator represents one of the most vibrant research fields for optical sensor due to its supreme capability in label-free, rapid, ultra-sensitive and ultra-selective detection. The 
sensing light is coupled into the resonator with resonance condition, and constrained by waveguide surface with an evanescent field exponentially decaying into the surrounding medium [11,12]. The resonant wavelength is therefore affected by the refractive index of the solution in contact with the waveguide surface. Generally, microring resonators for sensing have focused on proof-of-principle investigations, such as improving performance of resonator [13], measuring in buffer of streptavidin-biotin interactions [14], detecting of proteins or virus [15]. Besides demonstrating the ability to monitor in real-time for the chemical modification and biological molecules binding of the sensor surface [16], the microring resonator also demonstrated to monitor multiplexed molecular binding simultaneously [17]. Previous reports concerning the recognition of biomolecules using microring resonator are mainly based on immunoassay techniques $[18,19]$. However, these recognition elements are time-consuming, unstable, typically possess high cost and only work under physiological conditions. Therefore, a robust and cost-effective recognition element is needed.

Molecular imprinting stands out as a promising method to create artificial receptors with molecular recognition sites [20]. The template molecule, functional monomer, crosslinking agent and initiating agent are aggregated into polymers followed by removing the templates to form recognition cavities [21]. The achieved MIPs are robust, stable and reproducible compared with natural material [22], representing an ideal alternative to biomolecules and having been widely used in biosensors [23,24] and other areas [25]. Usually, MIPs are combined with sensor by two typical methods. The first is that the pre-made MIP particles are immobilized on the surface of a sensor by physical capture [26]. This method forms a thick film and suffers from low sensitivity. The second utilizes in-situ self-assembly of MIPs directly in the surface of the sensor. Ultrathin film can be obtained and better sensitivity has been demonstrated compared to physical capture $[27,28]$. Different sensor structures have been applied in the second approach [24,29].

In this study, MIPs were prepared in the surface of microring resonator to detect testosterone by thermal polymerization. The template molecule and functional monomer were firstly treated with pre-polymerization to form self-assembled monolayer (SAM). The initiating agent was then covalently coupled to the carboxyl-terminated SAM, followed by MIPs directly immobilized in surface of the sensor during polymerization. The fabricated MIPs sensor was used to detect different concentrations of testosterone. The selectivity of the MIPs sensor was also evaluated by detecting microcystin-LR. Subsequently, the sensor was regenerated by an acetic acid-ethanol solution. Ultrahigh sensitivity, good specificity and reproducibility have been demonstrated, promising for real-time and low-cost salivary testosterone detection.

\section{Experimental Section}

\subsection{Reagents}

Methacrylic acid (MAA), Ethylene glycol dimethacrylate (EGDMA), acetonitrile and acetic acid were purchased from Sigma-Aldrich (Schnelldorf, Germany). Testosterone and 2,2'-Azobisiobutyronitrile (AIBN) were purchased from Sinopharm Chemical Reagent Co. Ltd (Shanghai, China). All other reagents were purchased from Sinopharm Chemical Reagents Co. Ltd (Shanghai, China).

\subsection{Instrumentation and Microring Sensor}

The instrumentation to measure transmission spectrum of microring resonator contains a tunable light source (Agilent $81600 \mathrm{~B}$ ) coupled into input waveguide and a power meter (Agilent $81635 \mathrm{~A}$ ) to collect the output light. Sensor chips employing grating couplers were manufactured on silicon-on-insulator (SOI) wafers with a $220 \mathrm{~nm}$-thick top silicon layer and $2 \mu \mathrm{m}$-thick buried oxide layer, as shown in Figure 1. Figure 2a shows the optical microscope image of the microring resonator sensor and Figure $2 b$ shows the scanning electron microscopy (SEM) image of the waveguide. The 
radius of ring is $32 \mu \mathrm{m}$ and the length of directional coupler is $8 \mu \mathrm{m}$. In addition, the width of waveguide and gap are $500 \mathrm{~nm}$. All the strip waveguides are deep etched with $220 \mathrm{~nm}$ except grating couplers are shallow etched with $70 \mathrm{~nm}$. The whole sensor is coated by $\mathrm{SiO}_{2}$ with $2 \mu \mathrm{m}$-thick upper cladding layer while the microring resonator is exposed to the analyzed sample by removing the upper cladding layer in the sensing window.

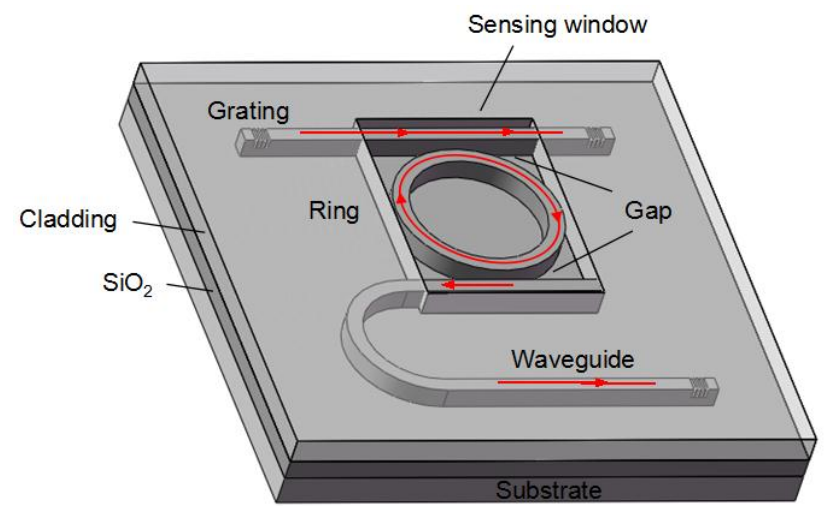

Figure 1. Schematic of the microring resonator sensor.
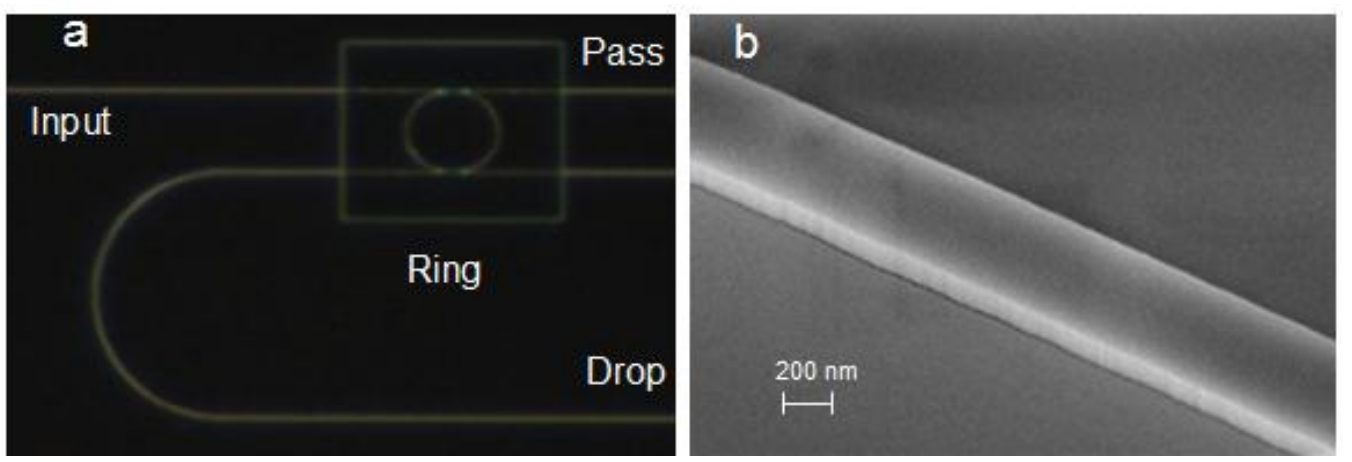

Figure 2. (a) Optical microscope image of the microring resonator; (b) SEM image of the waveguide.

\subsection{MIPs Synthesis}

At first, the sensor chip was cleaned by acetone, ethanol and deionized (DI) water for $5 \mathrm{~min}$ individually, then dried using nitrogen. As illustrated by the schematic in Figure 3, for the preparation of self-assembly, $5 \mathrm{mg}$ of testosterone and $400 \mu \mathrm{L}$ of functional monomer MAA were added into $2 \mathrm{~mL}$ of acetronitrile and placed at $25^{\circ} \mathrm{C}$ for $3 \mathrm{~h}$. Subsequently, $0.5 \mathrm{~mL}$ of crosslinking agent EGDMA and $9 \mathrm{mg}$ of initiating agent AIBN were appended to the solution. After being treated with nitrogen gas for $10 \mathrm{~min}$, the reaction solution was cast onto the surface of sensor chip. The sensor chip was placed in a hot-air oven at $60^{\circ} \mathrm{C}$ for $12 \mathrm{~h}$ for polymerization. After the polymerization, the sensor chip was washed by an acetic acid-ethanol solution (volume ratio is 1:1) to remove potential residual organics and the template molecule. At last, the sensor chip was rinsed by DI water and dried by nitrogen. Furthermore, non-imprinted polymer films (NIPs) were synthesized without using the testosterone as template molecule, and were used to evaluate the specificity of the sensor. 

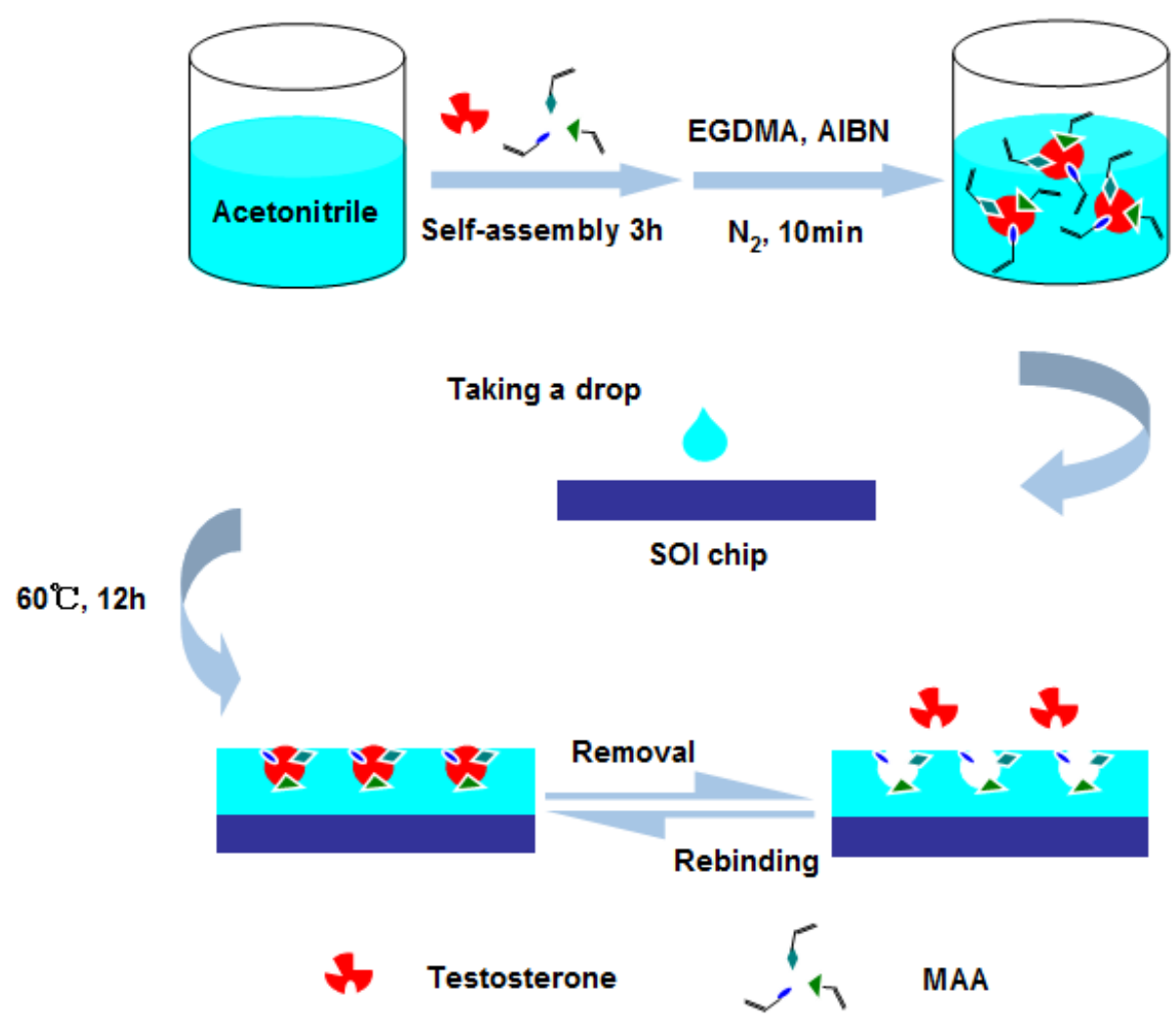

Figure 3. Schematic for preparation molecularly imprinted polymers on the surface of chip.

\subsection{Testosterone Detection}

The solution of testosterone is made by serial dilution in DI water. Before each measurement, the MIPs sensor was taken out and stabilized for several minutes. Then, the sensor chip is exposed to testosterone solution with different concentrations. The transmission spectrum for each solution is measured. The sensor chip is washed by an acetic acid-ethanol solution (volume ratio is 1:1), cleaned by DI water and dried by nitrogen after each measurement.

\section{Results and Discussion}

\subsection{Quantitative Detection}

The adsorption properties of sensor were characterized by the shift of resonant wavelength. The affinity binding of testosterone molecules was detected by a series of testosterone samples with the concentrations ranging from 0.05 to $10 \mathrm{ng} / \mathrm{mL}$. The transmission spectra and the wavelength shift of the sensor chips coated by the MIPs and NIPs respectively, in the different concentrations of testosterone were showed in Figure 4. From Figure 4a,b, a remarkable resonant wavelength shift of sensor coated by the MIPs can be clearly observed when changing the concentration of testosterone from $0.05 \mathrm{ng} / \mathrm{mL}$ to $0.2 \mathrm{ng} / \mathrm{mL}$, but the resonant wavelength of the sensor coated by the NIPs is not significantly shift. Furthermore, the sensitivity of sensor is $\mathrm{S}=4.803 \mathrm{~nm} / \mathrm{ng} \cdot \mathrm{ml}^{-1}$, which obtained by fitting as shown in Figure 4c. We adopt the traditional method of using 3 times standard deviations $\sigma$ as a measure of the sensor resolution [30] $\mathrm{R}=3 \sigma=0.234 \mathrm{~nm}$, while $\sigma=0.078 \mathrm{~nm}$ depends on the total system noise and the spectral resolution. Furthermore, the detection limit $\mathrm{L}=\mathrm{R} / \mathrm{S}=48.7 \mathrm{pg} / \mathrm{mL}$ were obtained. This result suggested that the microring resonator coated by MIPs can adsorb the template molecules and ultrahigh sensitivity. 

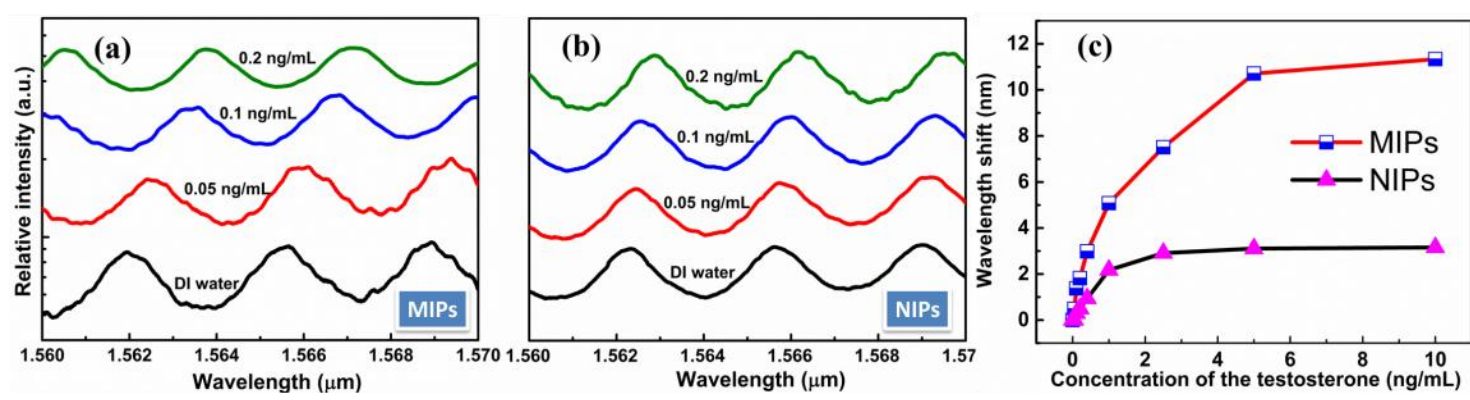

Figure 4. (a) Transmission spectra of molecularly imprinted polymer films (MIPs) when detecting testosterone; (b) Transmission spectra of non-imprinted polymer films (NIPs) when detecting testosterone; (c) Wavelength shift of MIPs and NIPs film coated chips, for the detection of different concentration of testosterone.

\subsection{Specific Recognition}

The microcystin-LR was used to evaluate the specificity of the MIPs coated chip response. As shown in Figure 5, results indicate that the sensor was only sensitive to testosterone, but not to microcystin-LR molecular. The results strongly demonstrated that the sensor had a good specific recognition for testosterone.

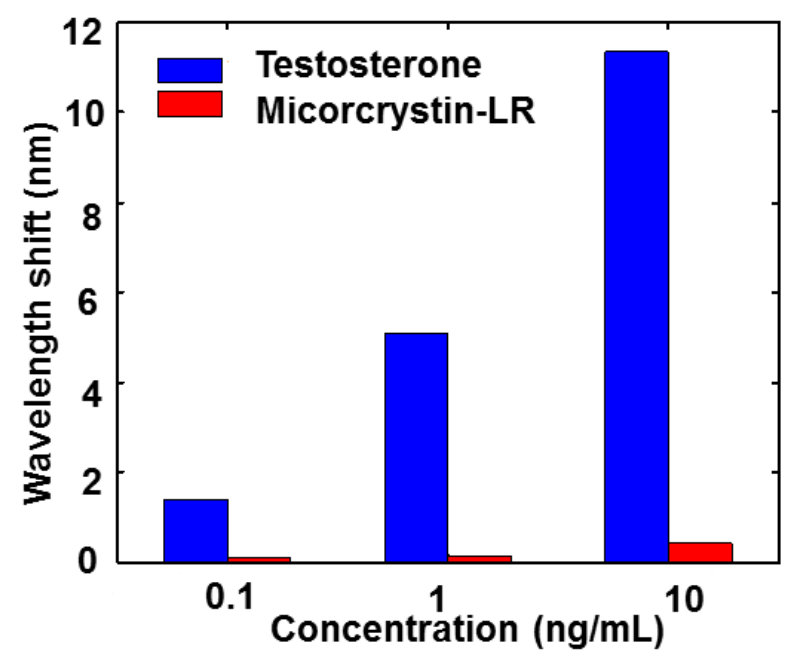

Figure 5. Wavelength shift of MIPs film coated chips, for the detection of different concentration of testosterone and microcystin-LR.

\subsection{Reproducibility}

To evaluate the reproducibility of the MIPs, the microring resonator chip was regenerated by rinsing in an acetic acid-ethanol solution (volume ratio is 1:1) and DI water, dried by nitrogen after each measurement. The transmission spectrum was re-measured in the same condition and the comparison of resonant wavelength shift was shown in Figure 6. The fabrication process of MIPs indicated that the MIPs can be regenerated many times. However, the response of the sensor has a drift for $1 \mathrm{ng} / \mathrm{mL}$ testosterone in the test of the reproducibility as shown in Figure 6 because the MIPs were damaged a little when testing. This limits the maximal number of the sensor regenerations. 


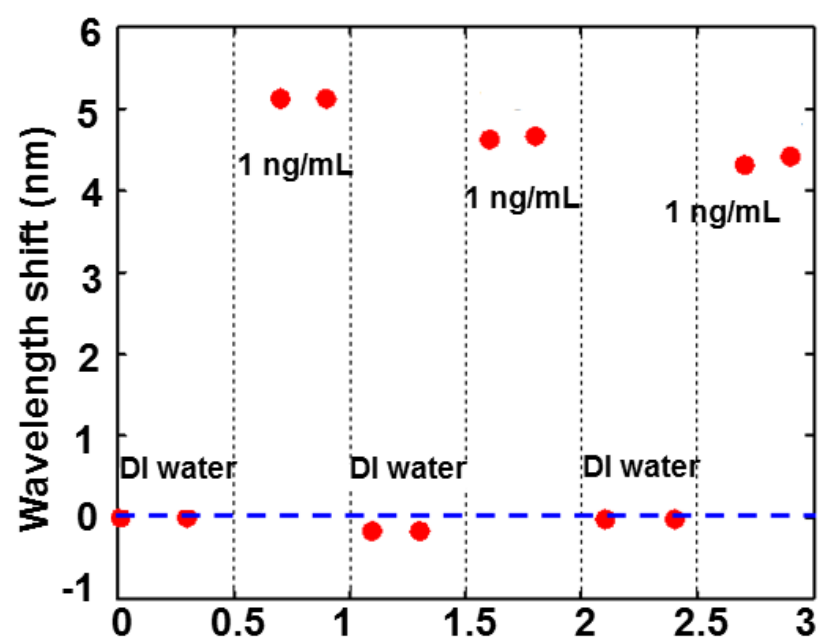

Figure 6. Resonant wavelength shift of the sensor for $1 \mathrm{ng} / \mathrm{mL}$ testosterone after each regeneration.

\section{Conclusions}

The reported biosensor has achieved a low detection limit of $48.7 \mathrm{pg} / \mathrm{mL}$ based on a combination of microring resonator and self-assembled MIPs. The MIPs can be easily grown on the sensor surface with simple procedures. Ultrahigh sensitivity, good specificity and reproducibility of the sensor have been demonstrated. This robust, stable and reproducible assay system is strategically important for salivary testosterone detection in near real-time clinical diagnostics.

Acknowledgments: This work was supported by National High Technology Research and Development Program of China (NO.2014AA06A504), Science and Technology Department of Zhejiang Province (NO.2014C31030), Fundamental Research Funds for Central Universities (NO.2014QNA5018), the National Natural Science Foundation of China (NO.61535010), Zhejiang Provincial Natural Science Foundation of China (NO.LY16F050001).

Author Contributions: Yangqing Chen, Wen-Fei Dong, Zhimin Chang, Xiaodan Shen and Yong Liu have been contributed for detecting idea, performing the experiments and analyzing the experimental results. Mingyu Li, Longhua Tang and Jian-Jun He are the project supervisors.

Conflicts of Interest: The authors declare no conflict of interest.

\section{References}

1. Inoue, K.; Ferrante, P.; Yu, H.; Yasukawa, T.; Shiku, H.; Matsue, T. A competitive immunochromatographic assay for testosterone based on electrochemical detection. Talanta 2007, 73, 886-892. [CrossRef] [PubMed]

2. Cooper, E.R.; Mcgrath, K.C.; AK, H. In vitro androgen bioassays as a detection method for designer androgens. Sensors 2013, 13, 2148-2163. [CrossRef] [PubMed]

3. West, D.W.D.; Phillips, S.M. Associations of exercise-induced hormone profiles and gains in strength and hypertrophy in a large cohort after weight training. Eur. J Appl. Physiol. 2012, 112, 2693-2702. [CrossRef] [PubMed]

4. Bos, P.A.; David, T.; Jack, V.H. Testosterone decreases trust in socially naive humans. Proc. Natl. Acad. Sci. USA 2010, 107, 9991-9995. [CrossRef] [PubMed]

5. Shrivastav, T.G.; Basu, A.; Kariya, K.P. One step enzyme linked immunosorbent assay for direct estimation of serum testosterone. J. Immunoass. Immunochem. 2003, 24, 205-217. [CrossRef] [PubMed]

6. Mohan, R.K.; Kadwad, V.; Samuel, G.; Venkatesh, M.; Sivaprasad, N. Solid phase radioimmunoassay for testosterone in human serum using antibodies coupled to magnetizable cellulose. J. Radioanal. Nucl. Chem. 2006, 268, 461-466. [CrossRef]

7. Coyotupa, J.; Parlow, A.F.; Abraham, G.E. Simultaneous radioimmunoassay of plasma testosterone and dihydrotestosterone. Anal. Lett. 1972, 5, 329-340. [CrossRef] 
8. Kalhorn, T.F.; Page, S.T.; Howald, W.N.; Mostaghel, E.A.; Nelson, P.S. Analysis of testosterone and dihydrotestosterone from biological fluids as the oxime derivatives using high-performance liquid chromatography/tandem mass spectrometry. Rapid Commun. Mass Spectrom. Rcm 2007, 21, 3200-3206. [CrossRef] [PubMed]

9. Taieb, J. Testosterone measured by 10 immunoassays and by isotope-dilution gas chromatography-mass spectrometry in sera from 116 men, women, and children. Clin. Chem. 2003, 49, 1381-1395. [CrossRef] [PubMed]

10. Cawood, M.L.; Field, H.P.; Ford, C.G.; Gillingwater, S.; Kicman, A.; Cowan, D.; Barth, J.H. Testosterone measurement by isotope-dilution liquid chromatography-tandem mass spectrometry: Validation of a method for routine clinical practice. Clin. Chem. 2005, 51, 1472-1479. [CrossRef] [PubMed]

11. Passaro, V.M.N.; Dell'Olio, F.; de Leonardis, F. Ammonia optical sensing by microring resonators. Sensors 2007, 7, 2741-2749. [CrossRef]

12. Passaro, V.M.N.; Dell'Olio, F.; Casamassima, B.; de Leonardis, F. Guided-wave optical biosensors. Sensors 2007, 7, 508-536. [CrossRef]

13. Ciminelli, C.; Dell'Olio, F.; Conteduca, D.; Campanella, C.M.; Armenise, M.N. High performance soi microring resonator for biochemical sensing. Opt. Laser Technol. 2014, 59, 60-67. [CrossRef]

14. Katrien, D.V.; Irene, B.; Etienne, S.; Peter, B.; Roel, B. Silicon-on-insulator microring resonator for sensitive and label-free biosensing. Opt. Expr. 2007, 15, 7610-7615.

15. Dell'Olio, F.; Ciminelli, C.; Armenise, M.N.; Conteduca, D. New ultrasensitive resonant photonic platform for label-free biosensing. Opt. Expr. 2015, 23, 28593-28604. [CrossRef] [PubMed]

16. Ciminelli, C.; Campanella, C.M.; Dell'Olio, F.; Campanella, C.E.; Armenise, M.N. Label-free optical resonant sensors for biochemical applications. Prog. Quantum Electron. 2013, 37, 51-107. [CrossRef]

17. Washburn, A.L.; Gomez, J.; Bailey, R.C. DNA-encoding to improve performance and allow parallel evaluation of the binding characteristics of multiple antibodies in a surface-bound immunoassay format. Anal. Chem. 2011, 83, 3572-3580. [CrossRef] [PubMed]

18. Mcclellan, M.S.; Domier, L.L.; Bailey, R.C. Label-free virus detection using silicon photonic microring resonators. Biosens. Bioelectron. 2012, 31, 388-392. [CrossRef] [PubMed]

19. Washburn, A.L.; Gunn, L.C.; Bailey, R.C.; Chem., A. Label-free quantitation of a cancer biomarker in complex media using silicon photonic microring resonators. Anal. Chem. 2009, 81, 9499-9506. [CrossRef] [PubMed]

20. Haupt, K.; Mosbach, K. Molecularly imprinted polymers and their use in biomimetic sensors. Chem. Rev. 2000, 100, 2495-2504. [CrossRef] [PubMed]

21. Bossi, A.; Bonini, F.; Turner, A.P.; Piletsky, S.A. Molecularly imprinted polymers for the recognition of proteins : The state of the art. Biosens. E Bioelectron. 2007, 22, 1131-1137.

22. Haupt, K.; Belmont, A.S. Molecularly imprinted polymers as recognition elements in sensors. Handb. Biosens. Biochips 2008, 2, 23-39.

23. Yola, M.L.; Uzun, L.; Özaltın, N.; Denizli, A. Development of molecular imprinted nanosensor for determination of tobramycin in pharmaceuticals and foods. Talanta 2014, 120, 318-324. [CrossRef] [PubMed]

24. Hao, H.; Zhou, L.; Yi, W.; Li, C.; Jia, Y.; Wei, Z.; Zhang, Q.; Li, M.; Li, H.; Dong, W.F. Detection of trace microcystin-LR on a $20 \mathrm{mhz}$ QCM sensor coated with in situ self-assembled MIPs. Talanta 2015, 131, 8-13.

25. Zhu, G.; Wang, X.; Gao, X.; Fan, J. Preparation of surface molecularly imprinted polymer and selective extraction of 1-methoxyethyl-3-methylimidazolium bis[(trifluoromethyl)sulfonyl]imide. Monatsh. für Chem./Chem. Mon. 2015, 146, 1-10. [CrossRef]

26. Gültekin, A.; Karanfil, G.; Kuş, M.; Sönmezoğlu, S.; Say, R. Preparation of MIP-based QCM nanosensor for detection of caffeic acid. Talanta 2014, 119, 533-537. [CrossRef] [PubMed]

27. Kugimiya, A.; Takeuchi, T. Surface plasmon resonance sensor using molecularly imprinted polymer for detection of sialic acid. Biosens. Bioelectron. 2001, 16, 1059-1062. [CrossRef]

28. Reimhult, K.; Yoshimatsu, K.; Risveden, K.; Si, C.; Lei, Y.; Krozer, A. Characterization of QCM sensor surfaces coated with molecularly imprinted nanoparticles. Biosens. Bioelectron. 2008, 23, 1908-1914. [CrossRef] [PubMed] 
29. Lotierzo, M.; Henry, O.Y.F.; Piletsky, S.; Tothill, I.; Cullen, D.; Kania, M.; Hock, B.; Turner, A.P.F. Surface plasmon resonance sensor for domoic acid based on grafted imprinted polymer. Biosens. Bioelectron. 2004, 20, 145-152. [CrossRef] [PubMed]

30. Carlborg, C.F.; Gylfason, K.B.; Kaźmierczak, A.; Dortu, F.; Polo, M.B.; Catala, A.M.; Kresbach, G.; Sohlström, H.; Moh, T.; Vivien, L. A packaged optical slot-waveguide ring resonator sensor array for multiplex label-free assays in labs-on-chips. Lab Chip 2010, 10, 281-290. [CrossRef] [PubMed]

(C) 2015 by the authors; licensee MDPI, Basel, Switzerland. This article is an open access article distributed under the terms and conditions of the Creative Commons by Attribution (CC-BY) license (http:/ / creativecommons.org/licenses/by/4.0/). 